\title{
Three extensions of Ćirić quasicontraction on partial metric spaces
}

\author{
Dejan Ilić, Vladimir Pavlović and Vladimir Rakočević*
}

"Correspondence: vrakoc@ptt.rs Department of Mathematics,

Faculty of Sciences and Mathematics, University of Nišs, Niš, 18000, Serbia

\begin{abstract}
In this paper we define and study three extensions of the notion of Cirić quasicontraction to the context of partial metric spaces. For such mappings, we prove fixed point theorems. Among other things, we generalize a recent result of Altun, Sola and Simsek, of Ilić et al., of Matthews, and the main result of Ćirić is also recovered. The theory is illustrated by some examples.
\end{abstract}

MSC: $15 \mathrm{~A} 09 ; 15 \mathrm{~A} 24$

Keywords: fixed point; partial metric space; quasicontraction

\section{Introduction and preliminaries}

Let $(X, \rho)$ be a complete metric space. A map $T: X \mapsto X$, such that for some constant $\lambda \in(0,1)$ and for every $x, y \in X$, there is the inequality

$$
\rho(T x, T y) \leq \lambda \cdot \max \{\rho(x, y), \rho(x, T x), \rho(y, T y), \rho(x, T y), \rho(y, T x)\},
$$

is called a quasicontraction. Let us remark that Cirić [1] (see also [2, 3]) introduced and studied quasicontractions as one of the most general types of contractive maps. The wellknown Ćirić result is that every quasicontraction $T$ possesses a unique fixed point.

There exist many generalizations of the concept of metric spaces in the literature. In particular, Matthews [4] introduced the notion of partial metric space as a part of the study of denotational semantics of dataflow networks, showing that the Banach contraction mapping theorem can be generalized to the partial metric context for applications in program verification. After that, fixed point results in partial metric spaces have been studied by many other authors.

References [5-31] are some works in this line of research. The existence of several connections between partial metrics and topological aspects of domain theory were pointed out in, e.g., [11, 24, 32-34].

In this paper we study fixed point results about certain extensions of the notion of Ćirić quasicontraction to the setting of partial metric spaces, and we give some generalized versions of the fixed point theorem of Matthews as well as the main result of Ilić et al. [35]. The theory is illustrated by some examples.

Throughout this paper, the letters $\mathbb{R}, \mathbb{R}^{+}, \mathbb{Q}, \mathbb{N}$ denote the sets of real numbers, nonnegative real numbers, rational numbers and positive integers, respectively.

Let us recall [4] that a mapping $p: X \times X \rightarrow \mathbb{R}^{+}$, where $X$ is a nonempty set, is said to be a partial metric on $X$ if for any $x, y, z \in X$, the following four conditions hold true:

@2013 llić et al.; licensee Springer. This is an Open Access article distributed under the terms of the Creative Commons Attribution License (http://creativecommons.org/licenses/by/2.0), which permits unrestricted use, distribution, and reproduction in any medium, provided the original work is properly cited. 
(P1) $x=y$ if and only if $p(x, x)=p(y, y)=p(x, y)$,

(P2) $p(x, x) \leq p(x, y)$,

(P3) $p(x, y)=p(y, x)$,

(P4) $p(x, z) \leq p(x, y)+p(y, z)-p(y, y)$.

The pair $(X, p)$ is then called a partial metric space. A sequence $\left\{x_{m}\right\}_{m=0}^{\infty}$ of elements of $X$ is called $p$-Cauchy if the $\operatorname{limit}_{m, n} p\left(x_{n}, x_{m}\right)$ exists and is finite. The partial metric space $(X, p)$ is called complete if for each $p$-Cauchy sequence $\left\{x_{m}\right\}_{m=0}^{\infty}$, there is some $z \in X$ such that

$$
p(z, z)=\lim _{n} p\left(z, x_{n}\right)=\lim _{n, m} p\left(x_{n}, x_{m}\right)
$$

If $(X, p)$ is a partial metric space, then $p^{s}(x, y)=2 p(x, y)-p(x, x)-p(y, y), x, y \in X$, is a metric on $X,\left\{x_{n}\right\}_{n \geq 1}$ converges to $z \in X$ with respect to $p^{s}$ if and only if (1.2) holds, and $(X, p)$ is a complete partial metric space if and only if $\left(X, p^{s}\right)$ is a complete metric space $($ see $[4,21])$.

A sequence $x_{n}$ in a partial metric space $(X, p)$ is called 0-Cauchy [27] if $\lim _{m, n} p\left(x_{n}\right.$, $\left.x_{m}\right)=0$. We say that $(X, p)$ is 0 -complete if every 0 -Cauchy sequence in $X$ converges, with respect to $p$, to a point $x \in X$ such that $p(x, x)=0$. Note that every 0 -Cauchy sequence in $(X, p)$ is Cauchy in $\left(X, p^{s}\right)$, and that every complete partial metric space is 0 complete. A paradigm for partial metric spaces is the pair $(X, p)$ where $X=\mathbb{Q} \cap[0,+\infty)$ and $p(x, y)=\max \{x, y\}$ for $x, y \geq 0$, which provides an example of a 0 -complete partial metric space which is not complete.

\section{Auxiliary results}

In this section we define three extensions of the notion of Ciric quasicontraction to the context partial metric spaces and establish a few auxiliary results that will be used in the next, main section.

Definition 2.1 Let $(X, p)$ be a partial metric space and $T: X \rightarrow X$ be a mapping. If for some $\alpha \in[0,1)$ and all $x, y \in X$, there holds

$$
p(T x, T y) \leq \max \{\alpha M(x, y), p(x, x), p(y, y)\}
$$

where $M(x, y):=\max \{p(x, T x), p(x, T y), p(x, y), p(T x, y), p(y, T y)\}$, then we shall say that $T$ is a $p_{1}$-quasicontraction. If for all $x, y \in X$, the stronger condition

$$
p(T x, T y) \leq \max \left\{\alpha M(x, y), \frac{p(x, x)+p(y, y)}{2}\right\}
$$

is satisfied, we shall call $T$ a $p_{2}$-quasicontraction. If the even stronger condition

$$
p(T x, T y) \leq \alpha M(x, y)
$$

holds for all $x, y \in X$, then we shall say that $T$ is a $p$-quasicontraction.

Lemma 2.1 Let $(X, p)$ be a partial metric space, $T: X \rightarrow X$ be a $p_{1}$-quasicontraction, $x \in X$ and $n, m$ and $k$ be integers such that $m \geq n \geq k \geq 1$. 
If $p\left(T^{n} x, T^{m} x\right)>p\left(T^{i} x, T^{i} x\right)$ for all $i \in\{n-k, \ldots, n-1\} \cup\{m-k, \ldots, m-1\}$, then for some $i_{0}, j_{0} \in\{n-k, \ldots, m\}$, there must hold

$$
p\left(T^{n} x, T^{m} x\right) \leq \alpha^{k} p\left(T^{i_{0}} x, T^{j_{0}} x\right) .
$$

Proof This is an easy induction on $k$. For $k=1$ this follows from (2.1) and the assumptions made. Now suppose that the assertion holds for some $k \geq 1$ and all $m \geq n \geq k$, and let $m, n \geq k+1$ be such that we have $p\left(T^{n} x, T^{m} x\right)>p\left(T^{i} x, T^{i} x\right)$ for all $i \in\{n-(k+1), \ldots, n-$ $1\} \cup\{m-(k+1), \ldots, m-1\}$.

From $p\left(T^{n} x, T^{m} x\right)>\max \left\{p\left(T^{n-1} x, T^{n-1} x\right), p\left(T^{m-1} x, T^{m-1} x\right)\right\}$ and (2.1) we see that there must hold $p\left(T^{n} x, T^{m} x\right) \leq \alpha p\left(T^{n_{1}} x, T^{m_{1}} x\right)$ for some $\left(n_{1}, m_{1}\right) \in\{(n-1, n),(m-1, m),(n-$ $1, m-1),(n-1, m),(n, m-1)\}$ if $n<m$, or for some $\left(n_{1}, m_{1}\right) \in\{(n-1, n),(n-1, n-1)\}$ if $n=m$; in either case, we have $m_{1} \geq n_{1} \geq n-1 \geq k$.

There cannot be any $i \in\left\{n_{1}-k, \ldots, n_{1}-1\right\} \cup\left\{m_{1}-k, \ldots, m_{1}-1\right\} \subseteq\{n-(k+1), \ldots, n-$ $1\} \cup\{m-(k+1), \ldots, m-1\}$ such that $p\left(T^{n_{1}} x, T^{m_{1}} x\right) \leq p\left(T^{i} x, T^{i} x\right)$ because this would imply $p\left(T^{n} x, T^{m} x\right) \leq p\left(T^{i} x, T^{i} x\right)$. Thus, by the induction hypothesis, there are $i_{0}, j_{0} \in$ $\left\{n_{1}-k, \ldots, m_{1}\right\} \subseteq\{n-(k+1), \ldots, m\}$ such that $p\left(T^{n_{1}} x, T^{m_{1}} x\right) \leq \alpha^{k} p\left(T^{i_{0}} x, T^{j_{0}} x\right)$, whence $p\left(T^{n} x, T^{m} x\right) \leq \alpha^{k+1} p\left(T^{i_{0}} x, T^{j_{0}} x\right)$.

Lemma 2.2 Let $(X, p)$ be a partial metric space, $T: X \rightarrow X$ be a $p_{1}$-quasicontraction, $x \in X$ and $N_{x}:=\frac{1}{(1-\alpha)} p(x, T x)+p(x, x)$. Then

(1) for all $i, j \geq 0$, there holds

$$
p\left(T^{i} x, T^{j} x\right) \leq N_{x}
$$

(2) if we put $S_{x}:=\sup _{i \geq 0} p\left(T^{i} x, T^{i} x\right)$, then for some $i \geq 0$ we have

$$
p\left(T^{i} x, T^{i} x\right)=S_{x}
$$

Proof (1) Fix $m \geq 1$ and put $d:=\max \left\{p\left(T^{i} x, T^{j} x\right): 0 \leq i, j \leq m\right\}$.

It is easy to see that there must be some $i \in\{0,1, \ldots, m\}$ with $d=p\left(x, T^{i} x\right)$. Indeed, take any $i, j \in\{0,1, \ldots, m\}$ such that $d=p\left(T^{i} x, T^{j} x\right)$. If $0 \in\{i, j\}$, we are done. Otherwise $i, j \geq 1$, so $p\left(T^{i} x, T^{j} x\right) \leq \max \left\{\alpha p\left(T^{i_{1}} x, T^{j_{1}} x\right), p\left(T^{i-1} x, T^{i-1} x\right), p\left(T^{j-1} x, T^{j-1} x\right)\right\}$ for some $i_{1}, j_{1} \in$ $\{0,1, \ldots, m\}$. Therefore either $d \leq \alpha p\left(T^{i_{1}} x, T^{j_{1}} x\right) \leq \alpha d$, i.e., $d=0=p\left(x, T^{k} x\right)$ for any $0 \leq$ $k \leq m$, or $d \leq \max \left\{p\left(T^{i-1} x, T^{i-1} x\right), p\left(T^{j-1} x, T^{j-1} x\right)\right\} \leq d$, thus $d \in\left\{p\left(x, T^{i-1} x\right), p\left(x, T^{j-1} x\right)\right\}$.

Now take any $i_{0} \in\{0,1, \ldots, m\}$ such that $d=p\left(x, T^{i_{0}} x\right)$. If $i_{0}=0$, then $d \leq N_{x}$ follows trivially. If $i_{0} \geq 1$, then for some $i_{1}, i_{2} \in\{0,1, \ldots, m\}$,

$$
\begin{aligned}
d & =p\left(x, T^{i_{0}} x\right) \leq p(x, T x)+p\left(T x, T^{i_{0}} x\right) \\
& \leq p(x, T x)+\max \left\{\alpha p\left(T^{i_{1}} x, T^{i_{2}} x\right), p(x, x), p\left(T^{i_{0}-1} x, T^{i_{0}-1} x\right)\right\},
\end{aligned}
$$

so $d \leq p(x, T x)+\max \left\{\alpha d, p\left(T^{l} x, T^{l} x\right)\right\}$, where $l$ is the least integer such that $0 \leq l \leq m$ and $p\left(T^{l} x, T^{l} x\right)=\max \left\{p\left(T^{i} x, T^{i} x\right): 0 \leq i \leq m\right\}$. So it must either be $d \leq \frac{d(x, T x)}{1-\alpha} \leq N_{x}$ or $d \leq p(x, T x)+p\left(T^{l} x, T^{l} x\right)$. The latter possibility in the case $l=0$ gives $d \leq N_{x}$ directly, and if $l \geq 1$, then, since the minimality of $l$ implies $p\left(T^{l} x, T^{l} x\right)>p\left(T^{l-1} x, T^{l-1} x\right)$, we must have for some $j_{1}, j_{2} \in\{l-1, l\}$ that $d \leq p(x, T x)+\alpha p\left(T^{j_{1}} x, T^{j_{2}} x\right) \leq p(x, T x)+\alpha d$ and again $d \leq N_{x}$. 
(2) By part (1) we have $S_{x}<\infty$. If $S_{x}=0$, then there is nothing to prove. Thus let $S_{x}>0$. Suppose to the contrary that for all $i \geq 0$,

$$
p\left(T^{i} x, T^{i} x\right)<S_{x}
$$

Take $k \in \mathbb{N}$ such that $\alpha^{k} N_{x}<\frac{S_{x}}{2}$ and $i_{0} \in \mathbb{N}$ such that $p\left(T^{i_{0}} x, T^{i_{0}} x\right)>\frac{S_{x}}{2}$. By (2.6) we may assume that $i_{0} \geq k$. Also by (2.6) there must be some $n \in \mathbb{N}$ such that $n>i_{0}$ and $p\left(T^{n} x, T^{n} x\right)>p\left(T^{i} x, T^{i} x\right)$ for all $0 \leq i \leq i_{0}$. Denote by $n_{0}$ the least such integer.

Since $p\left(T^{n_{0}} x, T^{n_{0}} x\right)>p\left(T^{i} x, T^{i} x\right)$ for all $0 \leq i<n_{0}$ and since $n_{0} \geq k$, Lemma 2.1 implies that for some $j_{1}, j_{2} \geq n_{0}-k$, we have $p\left(T^{n_{0}} x, T^{n_{0}} x\right) \leq \alpha^{k} p\left(T^{j_{1}} x, T^{j_{2}} x\right) \leq \alpha^{k} N_{x}<\frac{S_{x}}{2}$. But $\frac{S_{x}}{2}<p\left(T^{i_{0}} x, T^{i_{0}} x\right)<p\left(T^{n_{0}} x, T^{n_{0}} x\right)$, a contradiction.

Lemma 2.3 Let $(X, p)$ be a partial metric space, $T: X \rightarrow X$ be a $p_{1}$-quasicontraction, $x \in X$ and $r_{x}:=\lim \sup _{i} p\left(T^{i} x, T^{i} x\right)$. Then

$$
\lim _{n, m} p\left(T^{n} x, T^{m} x\right)=r_{x}
$$

Proof Notice first that $r_{x}<\infty$ by Lemma 2.2. The lemma easily follows from the following two claims:

(1) for each $\epsilon>0$, there is some $k \in \mathbb{N}$ such that for all $n, m \geq k$, we have

$$
p\left(T^{n} x, T^{m} x\right)<r_{x}+\epsilon
$$

and

(2) if $r_{x}>0$, then

$$
p\left(T^{i} x, T^{i} x\right) \geq p\left(T^{i+1} x, T^{i+1} x\right) \text { holds for all but finitely many } i \geq 0
$$

To prove (1), fix any $\epsilon>0$. From $r_{x}=\lim \sup _{i} p\left(T^{i} x, T^{i} x\right)<r_{x}+\epsilon$, we see that there is some $k_{0} \in \mathbb{N}$ such that $\alpha^{k_{0}} N_{x}<r_{x}+\epsilon$ and such that for all $i \geq k_{0}$ we have $p\left(T^{i} x, T^{i} x\right)<r_{x}+\epsilon$.

Let $m \geq n \geq 2 k_{0}$. If $p\left(T^{n} x, T^{m} x\right) \leq p\left(T^{i} x, T^{i} x\right)$ for some $i \geq k_{0}$, then $p\left(T^{n} x, T^{m} x\right)<r_{x}+\epsilon$. Otherwise, as $n-k_{0} \geq k_{0}$, by Lemma 2.1 there are $i_{0}, j_{0} \geq n-k_{0}$ such that $p\left(T^{n} x, T^{m} x\right) \leq$ $\alpha^{k_{0}} p\left(T^{i_{0}} x, T^{j 0} x\right) \leq \alpha^{k_{0}} N_{x}<r_{x}+\epsilon$.

To prove (2) suppose $r_{x}>0$. Take any positive $\epsilon<\frac{r_{x}(1-\alpha)}{\alpha}$ and let $k \in \mathbb{N}$ be as claimed to exist in (1). We will show that for any $n \geq k$, it must be that $p\left(T^{n} x, T^{n} x\right)=\max \left\{p\left(T^{i} x, T^{i} x\right) \mid\right.$ $i \geq n$ \}. Indeed, suppose that for some $n \geq k$ this were not true. By (2) of Lemma 2.2, there is an integer $l$ such that $l \geq n$ and $p\left(T^{l} x, T^{l} x\right)=\max \left\{p\left(T^{i} x, T^{i} x\right) \mid i \geq n\right\}$. Let $m$ be the least such integer. Then by our assumption $m-1 \geq n$, so, by the choice of $m$, we must have $p\left(T^{m} x, T^{m} x\right)>p\left(T^{m-1} x, T^{m-1} x\right)$. Hence $p\left(T^{m} x, T^{m} x\right) \leq \alpha p\left(T^{m-1} x, T^{m} x\right) \leq \alpha\left(r_{x}+\epsilon\right)$. Since by definition of $r_{x}$ and $m$ it must be $r_{x} \leq p\left(T^{m} x, T^{m} x\right)$, the last inequality now yields $r_{x} \leq \alpha\left(r_{x}+\epsilon\right)$, i.e., $\frac{r_{x}(1-\alpha)}{\alpha} \leq \epsilon$, a contradiction.

Lemma 2.4 Let $(X, p)$ be a partial metric space, $T: X \rightarrow X$ be a $p_{1}$-quasicontraction and $x, y \in X$ be such that

$$
\lim _{n, m} p\left(T^{n} x, T^{m} x\right)=\lim _{n} p\left(y, T^{n} x\right)=p(y, y)
$$

Then $p(y, y)=p(y, T y)$. 
Proof Denote $\delta_{n}:=p\left(y, T^{n} x\right)-p\left(T^{n} x, T^{n} x\right)$ and $\theta_{n}:=p\left(y, T^{n-1} x\right)-p(y, y)$ and notice that

$$
\begin{aligned}
p(y, T y) \leq & \delta_{n}+p\left(T^{n} x, T y\right) \\
\leq & \delta_{n}+\max \left\{\alpha p\left(T^{n-1} x, T y\right), \alpha p\left(T^{n} x, y\right), \alpha p\left(T^{n-1} x, y\right),\right. \\
& \left.\alpha p\left(T^{n-1} x, T^{n} x\right), \alpha p(y, T y), p\left(T^{n-1} x, T^{n-1} x\right), p(y, y)\right\}
\end{aligned}
$$

implies, using $p\left(T^{n-1} x, T y\right) \leq \theta_{n}+p(y, T y)$, that

$$
\begin{aligned}
p(y, T y) \leq & \frac{\delta_{n}}{(1-\alpha)}+\max \left\{\frac{\alpha}{(1-\alpha)} \theta_{n}, \alpha p\left(T^{n} x, y\right), \alpha p\left(T^{n-1} x, y\right), \alpha p\left(T^{n-1} x, T^{n} x\right)\right. \\
& \left.p\left(T^{n-1} x, T^{n-1} x\right), p(y, y)\right\} .
\end{aligned}
$$

Now simply take the limit as $n \rightarrow \infty$ in the above inequality.

\section{Main results}

In this paper we study three extensions of Ćirić quasicontraction to a partial metric space. For such mappings, we prove fixed point theorems. Among other things, we generalize a recent result of Altun, Sola and Simsek, and we give some generalized versions of the fixed point theorem of Matthews, and the main result of Cirić is also recovered. The theory is illustrated by some examples.

Theorem 3.1 Let $(X, p)$ be a complete partial metric space and $T: X \rightarrow X$ be a $p_{1^{-}}$ quasicontraction. Then

(1) for each $x \in X$, the sequence $\left\{T^{n} x\right\}_{n \geq 1}$ converges with respect to $p^{s}$ to some point $\dot{x}$ such that $p(\dot{x}, \dot{x})=p(\dot{x}, T \dot{x})$;

(2) there is some $u \in X$ such that $u=T u$ and $p(u, u)=\inf _{x \in X} p(\dot{x}, \dot{x})$.

Proof By Lemmas 2.3 and 2.4 and completeness of $(X, p)$, for each $x \in X$, there must be some $\dot{x} \in X$ with $p(\dot{x}, \dot{x})=p(\dot{x}, T \dot{x})$ such that

$$
p(\dot{x}, \dot{x})=\lim _{n} p\left(\dot{x}, T^{n} x\right)=\lim _{n, m} p\left(T^{n} x, T^{m} x\right)=r_{x}
$$

Also notice that we must have

$$
p\left(T^{n} x, T^{n} x\right) \geq r_{x} \quad \text { for all but finitely many } n
$$

Denote $I:=\inf _{x \in X} r_{x}=\inf _{x \in X} p(\dot{x}, \dot{x})$. Using the construction described bellow, we will find a specific $u \in X$ such that $p(u, u)=p(u, T u)=I$ and later on we prove that we must actually have $T u=u$ for that particular point $u$. This will complete the proof.

Use (2.7) and (3.1) to find for each $n \in \mathbb{N}$ some $x_{n} \in X$ and $i_{n} \in \mathbb{N}$ such that

$$
\begin{aligned}
& \left\{p\left(T^{i_{n}-1} x_{n}, T^{i_{n}-1} x_{n}\right), p\left(T^{i_{n}-1} x_{n}, T^{i_{n}} x_{n}\right)\right. \\
& \left.\quad p\left(T^{i_{n}} x_{n}, T^{i_{n}} x_{n}\right), p\left(T^{i_{n}} x_{n}, T^{i_{n}+1} x_{n}\right)\right\} \subseteq\left[I, I+\frac{1}{n}\right) .
\end{aligned}
$$


Set $\delta_{n, m}^{\prime}:=\left[p\left(T^{i_{n}} x_{n}, T^{i_{n}+1} x_{n}\right)-p\left(T^{i_{n}+1} x_{n}, T^{i_{n}+1} x_{n}\right)\right]+\left[p\left(T^{i_{m}} x_{m}, T^{i_{m}+1} x_{m}\right)-p\left(T^{i_{m}+1} x_{m}\right.\right.$, $\left.\left.T^{i_{m}+1} x_{m}\right)\right]$ and $\theta_{n}^{\prime}:=p\left(T^{i_{n}} x_{n}, T^{i_{n}+1} x_{n}\right)-p\left(T^{i_{n}} x_{n}, T^{i_{n}} x_{n}\right)$.

Now

$$
\begin{aligned}
p( & \left.T^{i_{n}} x_{n}, T^{i_{m}} x_{m}\right) \\
\leq & \delta_{n, m}^{\prime}+p\left(T^{i_{n}+1} x_{n}, T^{i_{m}+1} x_{m}\right) \\
\leq & \delta_{n, m}^{\prime}+\max \left\{\alpha p\left(T^{i_{n}} x_{n}, T^{i_{m}+1} x_{m}\right), \alpha p\left(T^{i_{n}+1} x_{n}, T^{i_{m}} x_{m}\right), \alpha p\left(T^{i_{n}} x_{n}, T^{i_{m}} x_{m}\right),\right. \\
& \left.\alpha p\left(T^{i_{n}} x_{n}, T^{i_{n}+1} x_{n}\right), \alpha p\left(T^{i_{m}} x_{m}, T^{i_{m}+1} x_{m}\right), p\left(T^{i_{n}} x_{n}, T^{i_{n}} x_{n}\right), p\left(T^{i_{m}} x_{m}, T^{i_{m}} x_{m}\right)\right\}
\end{aligned}
$$

gives, using $p\left(T^{i_{n}} x_{n}, T^{i_{m}+1} x_{m}\right) \leq p\left(T^{i_{n}} x_{n}, T^{i_{m}} x_{m}\right)+\theta_{m}^{\prime}$ and similarly $p\left(T^{i_{m}} x_{m}, T^{i_{n}+1} x_{n}\right) \leq$ $p\left(T^{i_{m}} x_{m}, T^{i_{n}} x_{n}+\theta_{n}^{\prime}\right)$

$$
\begin{aligned}
I & \leq p\left(T^{i_{n}} x_{n}, T^{i_{n}} x_{n}\right) \leq p\left(T^{i_{n}} x_{n}, T^{i_{m}} x_{m}\right) \\
& \leq \frac{\delta_{n, m}^{\prime}}{1-\alpha}+\max \left\{\frac{\alpha \theta_{m}^{\prime}}{1-\alpha}, \frac{\alpha \theta_{n}^{\prime}}{1-\alpha}, I+\frac{1}{\min \{n, m\}}\right\} .
\end{aligned}
$$

So $\lim _{n, m} p\left(T^{i_{n}} x_{n}, T^{i_{m}} x_{m}\right)=I$, and thus there is some $u \in X$ such that

$$
p(u, u)=\lim _{n, m} p\left(T^{i_{n}} x_{n}, T^{i_{m}} x_{m}\right)=\lim _{n} p\left(u, T^{i_{n}} x_{n}\right)=I=\inf _{x \in X} r_{x} .
$$

Let us show that

$$
p(u, u)=p(u, T u) .
$$

Set $\lambda_{n}:=p\left(u, T^{i_{n}} x_{n}\right)-p\left(T^{i_{n}} x_{n}, T^{i_{n}} x_{n}\right)$ and $\mu_{n}:=p\left(u, T^{i_{n}-1} x_{n}\right)-p(u, u)$. From

$$
\begin{aligned}
p(u, T u) \leq & \lambda_{n}+p\left(T^{i_{n}} x_{n}, T u\right) \\
\leq & \lambda_{n}+\max \left\{\alpha p\left(T^{i_{n}-1} x_{n}, T u\right), \alpha p\left(T^{i_{n}} x_{n}, u\right), \alpha p\left(T^{i_{n}-1} x_{n}, u\right),\right. \\
& \left.\alpha p\left(T^{i_{n}-1} x_{n}, T^{i_{n}} x_{n}\right), \alpha p(u, T u), p\left(T^{i_{n}-1} x_{n}, T^{i_{n}-1} x_{n}\right), p(u, u)\right\}
\end{aligned}
$$

it follows, using $p\left(T^{i_{n}-1} x_{n}, T u\right) \leq p(u, T u)+\mu_{n}$, that

$$
\begin{aligned}
p(u, T u) \leq & \frac{\lambda_{n}}{1-\alpha}+\max \left\{\frac{\alpha \mu_{n}}{1-\alpha}, \alpha p\left(T^{i_{n}} x_{n}, u\right), \alpha p\left(T^{i_{n}-1} x_{n}, u\right),\right. \\
& \left.\alpha p\left(T^{i_{n}-1} x_{n}, T^{i_{n}} x_{n}\right), p\left(T^{i_{n}-1} x_{n}, T^{i_{n}-1} x_{n}\right), p(u, u)\right\},
\end{aligned}
$$

so we must have $p(u, T u) \leq p(u, u)$.

We now prove by induction on $n \in \mathbb{N} \cup\{0\}$ that

$$
\lim _{k} p\left(T^{i_{k}} x_{k}, T^{n} u\right)=p(u, u)=I .
$$

For $n=0$, this is just (3.3). 
Now suppose that (3.5) holds for some $n \geq 0$. We have

$$
\begin{aligned}
I \leq & p\left(T^{i_{m}} x_{m}, T^{n+1} u\right) \\
\leq & \max \left\{\alpha p\left(T^{i_{m}-1} x_{m}, T^{n+1} u\right), \alpha p\left(T^{i_{m}} x_{m}, T^{n} u\right), \alpha p\left(T^{i_{m}-1} x_{m}, T^{n} u\right),\right. \\
& \left.\alpha p\left(T^{i_{m}-1} x_{m}, T^{i_{m}} x_{m}\right), \alpha p\left(T^{n} u, T^{n+1} u\right), p\left(T^{i_{m}-1} x_{m}, T^{i_{m}-1} x_{m}\right), p\left(T^{n} u, T^{n} u\right)\right\} .
\end{aligned}
$$

From this, using

$$
\begin{aligned}
p\left(T^{i_{m}-1} x_{m}, T^{n+1} u\right) & \leq p\left(T^{i_{m}} x_{m}, T^{n+1} u\right)+\left[p\left(T^{i_{m}} x_{m}, T^{i_{m}-1} x_{m}\right)-p\left(T^{i_{m}} x_{m}, T^{i_{m}} x_{m}\right)\right] \\
& \leq p\left(T^{i_{m}} x_{m}, T^{n+1} u\right)+\frac{1}{m} \quad \text { (here we recall (3.2)) }
\end{aligned}
$$

and

$$
\begin{aligned}
p\left(T^{i_{m}-1} x_{m}, T^{n} u\right) & \leq p\left(T^{i_{m}} x_{m}, T^{n} u\right)+\left[p\left(T^{i_{m}} x_{m}, T^{i_{m}-1} x_{m}\right)-p\left(T^{i_{m}} x_{m}, T^{i_{m}} x_{m}\right)\right] \\
& \leq p\left(T^{i_{m}} x_{m}, T^{n} u\right)+\frac{1}{m}
\end{aligned}
$$

and

$$
p\left(T^{n} u, T^{n+1} u\right) \leq p\left(T^{i_{m}} x_{m}, T^{n+1} u\right)+\left[p\left(T^{n} u, T^{i_{m}} x_{m}\right)-p\left(T^{i_{m}} x_{m}, T^{i_{m}} x_{m}\right)\right]
$$

and

$$
\begin{gathered}
p\left(T^{n} u, T^{n} u\right) \leq p(u, u), \quad \text { which follows after taking the limit as } k \rightarrow \infty \text { in } \\
p\left(T^{n} u, T^{n} u\right) \leq 2 p\left(T^{n} u, T^{i_{k}} x_{k}\right)-p\left(T^{i_{k}} x_{k}, T^{i_{k}} x_{k}\right),
\end{gathered}
$$

we deduce that

$$
\begin{aligned}
I \leq & p\left(T^{i_{m}} x_{m}, T^{n+1} u\right) \\
\leq & \max \left\{\frac{\alpha}{(1-\alpha) m}, \alpha\left[p\left(T^{i_{m}} x_{m}, T^{n} u\right)+\frac{1}{m}\right],\right. \\
& \left.\frac{\alpha}{(1-\alpha)}\left[p\left(T^{i_{m}} x_{m}, T^{n} u\right)-p\left(T^{i_{m}} x_{m}, T^{i_{m}} x_{m}\right)\right], I+\frac{1}{m}, p(u, u)\right\} .
\end{aligned}
$$

The last inequality, in view of the induction hypothesis and (3.3), immediately gives

$$
\lim _{m} p\left(T^{i_{m}} x_{m}, T^{n+1} u\right)=p(u, u)=I .
$$

We finally prove $T u=u$. By (3.4) we only need to show $p(T u, T u)=p(u, u)$. For a proof by contradiction, assume this is not the case. Then $p(T u, T u)<p(u, u)=I$.

Set $y:=T u$. By (3.1) and $r_{y} \geq I$, there must be some $j \geq 0$ such that $p\left(T^{j} y, T^{j} y\right) \geq I$. Let $m$ be the least such $j$. By our assumption, we must have $m \geq 1$. Then $p\left(T^{j} y, T^{j} y\right)<$ $p\left(T^{m} y, T^{m} y\right)$ for all $0 \leq j<m$. Thus by Lemma 2.1 there must be some $i, j \in\{0,1, \ldots, m\}$ such that $p\left(T^{m} y, T^{m} y\right) \leq \alpha^{m} p\left(T^{i} y, T^{j} y\right)$. So

$$
I \leq p\left(T^{m} y, T^{m} y\right) \leq \alpha^{m} p\left(T^{i+1} u, T^{j+1} u\right) .
$$


We have $p\left(T^{i+1} u, T^{j+1} u\right) \leq I$, which follows by (3.3) and (3.5) after taking the limit as $n \rightarrow \infty$ in $p\left(T^{i+1} u, T^{j+1} u\right) \leq p\left(T^{i+1} u, T^{i_{n}} x_{n}\right)+p\left(T^{i_{n}} x_{n}, T^{j+1} u\right)-p\left(T^{i_{n}} x_{n}, T^{i_{n}} x_{n}\right)$. But $m \geq 1$ now yields $I \leq \alpha I$, i.e., $0=I>p(T u, T u)$, a contradiction.

Theorem 3.2 Let $(X, p)$ be a complete partial metric space and $T: X \rightarrow X$ be a $p_{2}$ quasicontraction. Then there is a unique fixed point $u \in X$ of $T$. For each $x \in X$, the sequence $\left\{T^{n} x\right\}_{n \geq 1}$ converges with respect to $p^{s}$ to some point $\dot{x}$ such that $p(\dot{x}, \dot{x})=p(\dot{x}, T \dot{x})$, and there is the equality $p(u, u)=\inf _{x \in X} p(\dot{x}, \dot{x})$.

Proof By Theorem 3.1 it remains to verify the uniqueness of the fixed point, so let $u, v \in X$ be such that $T u=u$ and $T v=v$. By (2.2) we have

$$
p(u, v)=p(T u, T v) \leq \max \left\{\alpha p(u, v), \frac{p(u, u)+p(v, v)}{2}\right\} .
$$

Hence either $p(u, v)=0$ or $0 \geq 2 p(u, v)-p(u, u)-p(v, v)=p^{s}(u, v)$ and the assertion follows.

Theorem 3.3 Let $(X, p)$ be a 0-complete partial metric space and $T: X \rightarrow X$ be a $p$ quasicontraction. Then there is a unique fixed point $u \in X$ of $T$. Furthermore, we have $p(u, u)=0$ and for each $x \in X$ the sequence $\left\{T^{n} x\right\}_{n \geq 1}$ converges to $u$ with respect to $p^{s}$.

Proof By Lemma 2.3 we have $\lim _{n, m} p\left(T^{n} x, T^{m} x\right)=r_{x}$. Also $r_{x}<\infty$ by Lemma 2.2.

If we had $r_{x}>0$, then there would be some positive $\epsilon<\frac{r_{x}(1-\alpha)}{1+\alpha}$ and $n \in \mathbb{N}$ such that $p\left(T^{i} x, T^{j} x\right) \in\left(r_{x}-\epsilon, r_{x}+\epsilon\right)$ for all $i, j \in\{n-1, n\}$. Thus by (2.3) there would be some $i, j \in\{n-1, n\}$ such that

$$
r_{x}-\epsilon<p\left(T^{n} x, T^{n} x\right) \leq \alpha p\left(T^{i} x, T^{j} x\right) \leq \alpha\left(r_{x}+\epsilon\right)
$$

i.e., $\frac{r_{x}(1-\alpha)}{1+\alpha}<\epsilon$, a contradiction.

So $r_{x}=0$ and thus by 0 -completeness of $(X, p)$ there is some $u \in X$ such that $\lim _{n, m} p\left(T^{n} x\right.$, $\left.T^{m} x\right)=\lim _{n} p\left(u, T^{n} x\right)=p(u, u)=0$. But by Lemma 2.4 we have $p(u, T u)=p(u, u)=0$ so $T u=u$. The argument for uniqueness of the fixed point is standard.

Remark 3.1 Recently a very interesting paper by Haghi, Rezapour and Shahzad [36] showed up in which the authors associated to each partial metric space $(X, p)$ a metric space $(X, d)$ by setting $d(x, x)=0$ and $d(x, y)=p(x, y)$ if $x \neq y$ and proved that $(X, p)$ is 0 -complete if and only if $(X, d)$ is complete. They then proceeded to demonstrate how using the associated metric $d$ some of the fixed point results in partial metric spaces can easily be deduced from the corresponding known results in metric spaces.

Let us point out that these considerations can apply neither to $p_{1}$-quasicontractions nor to $p_{2}$-quasicontractions, since the terms $p(x, x)$ and $p(y, y)$ on the right-hand side of (2.1) and $\frac{1}{2}(p(x, x)+p(y, y))$ on the right-hand side of (2.2) do not get multiplied by $\alpha$. Thus Theorems 3.1 and 3.2 cannot follow from the result of Ćirić they generalize.

On the other hand, using the approach of Haghi, Rezapour and Shahzad, we now show how Theorem 3.3 can be directly deduced from Ćirićs result [1]. 
Given the assumptions of Theorem 3.3, let $d$ be defined as said above. So $(X, d)$ is a complete metric space (see Proposition 2.1 of [36]). Observe that we have $d(x, y) \leq p(x, y)$ for all $x, y \in X$. For $x, y \in X$, set

$$
\mathrm{S}(x, y)=\{(x, y),(x, T x),(x, T y),(y, T y),(y, T x)\}
$$

and also $\mathrm{M}_{p}(x, y)=\max \{p(a, b) \mid(a, b) \in \mathrm{S}(x, y)\}$ and $\mathrm{M}_{d}(x, y)=\max \{d(a, b) \mid(a, b) \in$ $\mathrm{S}(x, y)\}$. We thus have that

$$
p(T x, T y) \leq \alpha \mathrm{M}_{p}(x, y)
$$

for all $x, y \in X$. We check that for all $x, y \in X$ it holds $d(T x, T y) \leq \alpha \mathrm{M}_{d}(x, y)$, so that the main result from [1] can immediately be applied. Since in the case $x=y$ the inequality trivially holds, suppose $x \neq y$. So $p(x, y)=d(x, y)$.

Since $d(T x, T y) \leq p(T x, T y) \leq \alpha \mathrm{M}_{p}(x, y)$, it suffices to show that $\mathrm{M}_{p}(x, y) \leq \mathrm{M}_{d}(x, y)$. Let $(c, e) \in \mathrm{S}(x, y)$ be such that $\mathrm{M}_{p}(x, y)=p(c, e)$. If $c \neq e$, then $\mathrm{M}_{p}(x, y)=p(c, e)=d(c, e) \leq$ $\mathrm{M}_{d}(x, y)$. If $c=e$, then, since $c \in\{x, y\}$, it follows that $\mathrm{M}_{p}(x, y)=p(c, c) \leq \max \{p(x, x)$, $p(y, y)\} \leq p(x, y)=d(x, y) \leq \mathrm{M}_{d}(x, y)$.

Remark 3.2 Even though the results of Haghi et al. can deduce the same fixed point as the corresponding partial metric fixed point result, using the partial metric version computers evaluate faster since many nonsense terms are omitted. This is very important in computer science due to its cost and explains the vast body of partial metric fixed point results found in literature.

Now we give corollaries of the above theorems.

Corollary 3.1 ([35]) Let $(X, p)$ be a complete partial metric space, $\alpha \in[0,1)$ and $T: X \rightarrow X$ be a given mapping. Suppose that for each $x, y \in X$, the following condition holds:

$$
p(T x, T y) \leq \max \{\alpha p(x, y), p(x, x), p(y, y)\}
$$

Then

(1) the set $X_{p}:=\left\{x \in X \mid p(x, x)=\inf _{y \in X} p(y, y)\right\}$ is nonempty;

(2) there is a unique $u \in X_{p}$ such that $T u=u$;

(3) for each $x \in X_{p}$, the sequence $\left\{T^{n} x\right\}_{n \geq 1}$ converges with respect to the metric $p^{s}$ to $u$.

Proof Put $\rho_{p}:=\inf _{x \in X} p(x, x)$ and $I:=\inf _{x \in X} p(\dot{x}, \dot{x})$. Note that (3.6) implies $p(T x, T x) \leq$ $p(x, x)$ for all $x \in X$. So, from $p(\dot{x}, \dot{x})=\lim _{n} p\left(T^{n} x, T^{n} x\right)$ and $p\left(T^{n+1} x, T^{n+1} x\right) \leq p\left(T^{n} x, T^{n} x\right)$, $n \geq 0$, we see that $p(\dot{x}, \dot{x}) \leq p\left(T^{0} x, T^{0} x\right)=p(x, x)$. Thus $\rho_{p}=I$. By Theorem 3.1 , there is some $u \in X$ with $T u=u$ and $p(u, u)=\rho_{p}$. This means that $u \in X_{p} \neq \emptyset$. Now let $x \in X_{p}$ be arbitrary. Since $\rho_{p} \leq p(\dot{x}, \dot{x}) \leq p\left(T^{n} x, T^{n} x\right) \leq p(x, x)=\rho_{p}$, for all $n \in \mathbb{N}$, we have $p(\dot{x}, \dot{x})=$ $p\left(T^{n} x, T^{n} x\right)=\rho_{p}=p(u, u)$, and so $\lim _{n, m} p\left(T^{n} x, T^{m} x\right)=p(u, u)$. Also, from $p\left(u, T^{n+1} x\right) \leq$ $\left\{\alpha p\left(u, T^{n} x\right), \rho_{p}\right\}$ using $p\left(u, T^{n} x\right) \leq p\left(u, T^{n+1} x\right)+p\left(T^{n+1} x, T^{n} x\right)-p\left(T^{n+1} x, T^{n+1} x\right)$, it follows

$$
\rho_{p} \leq p\left(u, T^{n+1} x\right) \leq\left\{\frac{\alpha}{1-\alpha}\left[p\left(T^{n+1} x, T^{n} x\right)-p\left(T^{n+1} x, T^{n+1} x\right)\right], \rho_{p}\right\} .
$$

Hence $\lim _{n} p\left(u, T^{n} x\right)=\rho_{p}=p(u, u)$. 
Finally, if $v \in X_{p}$ is a fixed point, then by the preceding discussion $p(u, u)=\lim _{n} p\left(T^{n} v\right.$, $\left.T^{n} v\right)=\lim _{n} p\left(u, T^{n} v\right)$, i.e., $p(u, u)=p(v, v)=p(u, v)$ so $u=v$.

Corollary 3.2 Let $(X, p)$ be a complete partial metric space, $\alpha \in[0,1)$ and $T: X \rightarrow X$ be a given mapping. Suppose that for each $x, y \in X$, the following condition holds:

$$
p(T x, T y) \leq \max \left\{\alpha p(x, y), \frac{p(x, x)+p(y, y)}{2}\right\} .
$$

Then there is a unique $z \in X$ such that $T z=z$. Furthermore, $z \in X_{p}$ and for each $x \in X_{p}$, the sequence $\left\{T^{n} x\right\}_{n \geq 1}$ converges with respect to the metric $p^{s}$ to $z$.

As a corollary we obtain the already mentioned result of Matthews (see also Corollary 2 of [8] and [21]). Let us remark that the result of Matthews is for a complete partial metric space, but it is true for a 0 -complete partial metric space.

Corollary 3.3 (Matthews [4]) Let $(X, p)$ be a 0 -complete partial metric space, $\alpha \in[0,1)$ and $T: X \rightarrow X$ be a given mapping. Suppose that for each $x, y \in X$, the following condition holds:

$$
p(T x, T y) \leq \alpha p(x, y) .
$$

Then there is a unique $z \in X$ such that $T z=z$. Also $p(z, z)=0$ and for each $x \in X$ the sequence $\left\{T^{n} x\right\}_{n \geq 1}$ converges with respect to the metric $p^{s}$ to $z$.

Remark 3.3 In the case $p=\rho$ is a metric, by Theorem 3.3, the main result of Ćirić [1] is recovered. Theorem 3.3 also implies Corollaries 1-4 of [8], and the next Hardy and Rogers type [37] fixed point result. This result, under some extra conditions, was proved as one of the main results, Theorem 2 of [8].

Corollary 3.4 Let $(X, p)$ be a 0-complete partial metric space, $a, b, c, d, e \geq 0,0 \leq a+b+$ $c+d+e<1$, and $T: X \rightarrow X$ be a given mapping. Suppose that for each $x, y \in X$, the following condition holds:

$$
p(T x, T y) \leq a p(x, y)+b p(x, T x)+c p(x, T y)+d p(T x, y)+e p(y, T y) .
$$

Then there is a unique $z \in X$ such that $T z=z$. Also $p(z, z)=0$ and for each $x \in X$ the sequence $\left\{T^{n} x\right\}_{n \geq 1}$ converges with respect to the metric $p^{s}$ to $z$.

Example 3.1 Let $X:=[0,1] \cup[2,3]$ and define $p: X^{2} \rightarrow \mathbb{R}$ by

$$
p(x, y)= \begin{cases}\max \{x, y\}, & \{x, y\} \cap[2,3] \neq \emptyset, \\ |x-y|, & \{x, y\} \subseteq[0,1] .\end{cases}
$$

Then $(X, p)$ is a complete partial metric space. Define $T: X \rightarrow X$ by

$$
T x= \begin{cases}\frac{x+1}{2}, & 0 \leq x \leq 1, \\ 1, & x=2, \\ \frac{2+x}{2}, & 2<x \leq 3 .\end{cases}
$$


If $2<x \leq y \leq 3$, then it is easy to see that $p(T x, T y)=\frac{2+y}{2}$ and $M(x, y)=y$. Given any $\lambda \in[0,1)$, we have that $p(T x, T y)>\lambda M(x, y)$ holds for all $2<x \leq y \leq 3$ if $\lambda \leq \frac{1}{2}$, i.e., for all $2<x \leq y<\frac{2}{2 \lambda-1}$ if $\lambda>\frac{1}{2}$. On the other hand, we have that

$$
p(T x, T y) \leq \frac{1}{2} p(x, y), \quad\{x, y\} \subseteq[0,1]
$$

and

$$
p(T x, T y) \leq \frac{p(x, x)+p(y, y)}{2}, \quad\{x, y\} \cap[2,3] \neq \emptyset
$$

Thus $T$ is a $p_{2}$-quasicontraction on $X$ which is not a $p$-quasicontraction. By Theorem 3.2, there is a unique fixed point $z=1$. Also we have $p(1,1)=0=\min \{p(x, x) \mid x \in X\}$.

Example 3.2 Denote by $X_{\infty}$ the set of all sequences $x: \mathbb{N} \rightarrow \mathbb{N}$ and for $n \in \mathbb{N}$ by $X_{n}$ the set of all $n$-tuples $x:\{1, \ldots, n\} \rightarrow \mathbb{N}$ of positive integers. Put $X:=X_{\infty} \cup \bigcup_{n \in \mathbb{N}} X_{n}$. For $x, y \in X$, set

$$
I(x, y)=\{i \in \mathbb{N} \cup\{0\} \mid[j \in \operatorname{dom}(x) \cap \operatorname{dom}(y) \wedge j \leq i] \Rightarrow x(j)=y(j)\}
$$

and define $p(x, y):=\inf \left\{\frac{1}{2^{i}} \mid i \in I(x, y)\right\}$ (thus if $x(1) \neq y(1)$, then $I(x, y)=\{0\}$ and $\left.p(x, y)=1\right)$. Here ' $\operatorname{dom}(x)$ ' stands for the domain of the function $x$. Then $(X, p)$ is a partial metric space (see [4]) and a complete one as can easily be verified.

Define $T: X \rightarrow X$ by $T x=y$, where:

- $x \in X_{\infty} \Rightarrow y \in X_{\infty}$ and $x \in X_{n} \Rightarrow y \in X_{n+1}$;

- $y(1)=1, i \in \operatorname{dom}(x) \backslash\{1\} \Rightarrow y(i)=\min \{x(i), x(i-1)\}$ (this condition is vacuous if $x \in X_{1}$ ) and if in addition $x \in X_{n}$, then $y(n+1)=1$.

Note that taking, e.g., $x=(3,3,1)$ and $y=(3,3,2)$, we have $T x=(1,3,1,1)$ and $T y=$ $(1,3,2,1)$ so $p(T x, T y)=\frac{1}{4}=p(x, y)>\frac{1}{8}=p(x, x)=p(y, y)$. Thus the contractive condition of Corollary 3.1 is not satisfied. Nevertheless, there is a unique fixed point of $T$ - the sequence $s_{\infty}: \mathbb{N} \rightarrow \mathbb{N}$ defined by $s_{\infty}(i)=1$ for all $i \in \mathbb{N}$. We will show that $T$ is a $p$-quasicontraction. Consider arbitrary $x, y \in X$.

Case 1 . There is a nonnegative integer $i$ with $i+1 \in \operatorname{dom}(x) \cap \operatorname{dom}(y)$ such that $x(i+1) \neq$ $1 \vee y(i+1) \neq 1$. Denote by $k$ the least such nonnegative integer. Thus $k=0$ simply means that $x(1) \neq 1 \vee y(1) \neq 1$ and if $k \geq 1$, then for all $i \in \mathbb{N}$ with $i \leq k$, we must have $x(i)=y(i)=1$.

If $x(k+1) \neq y(k+1)$, then $p(x, y)=\frac{1}{2^{k}}$ so $p(T x, T y) \leq \frac{1}{2^{k+1}}=\frac{1}{2} p(x, y)$.

If $x(k+1)=y(k+1)$, then $p(x, T x)=\frac{1}{2^{k}}$ (because in this case we must have $x(k+1)=$ $y(k+1) \neq 1$ but $(T x)(k+1)=1)$. Hence $p(T x, T y) \leq \frac{1}{2^{k+1}}=\frac{1}{2} p(x, T x)$.

Case 2. $x(i)=1$ for all $i \in \operatorname{dom}(x)$ and $x \subseteq y$ (meaning $\operatorname{dom}(x) \subseteq \operatorname{dom}(y)$ and $x$ is the restriction of $y$ to the set $\operatorname{dom}(x))$.

If $x \in X_{n}$, then $p(T x, T y)=\frac{1}{2^{n+1}}=\frac{1}{2} p(x, y)$.

If $x \in X_{\infty}$, then $x=y=s_{\infty}=T x$, and so $p(T x, T y)=0$.

Case 3. $y(i)=1$ for all $i \in \operatorname{dom}(y)$ and $y \subseteq x$. This reduces to the previous case.

To illustrate the role condition (2.3) plays in ensuring the uniqueness of the fixed point, we modify a bit the definition of the operator $T$ to obtain the operator $T_{1}: X \rightarrow X$ determined by $T_{1} x=y$ if and only if

- $\operatorname{dom}(y)=\operatorname{dom}(x)$;

- $y(1)=1$ and $i \in \operatorname{dom}(x) \backslash\{1\} \Rightarrow y(i)=\min \{x(i), x(i-1)\}$. 
$T_{1}$ has infinitely many fixed points - these are exactly the sequence $s_{\infty}$ and the restrictions $s_{n}=(1, \ldots, n)$ of $s_{\infty}$ to the sets $\{1, \ldots, n\}$. Due to the existence of infinitely many fixed points, $T_{1}$ cannot be a $p_{2}$-quasicontraction. We verify that $T_{1}$ is a $p_{1}$-quasicontraction.

Given arbitrary $x, y \in X$, we distinguish three cases exactly as we did with the operator $T$.

Case 1 . There is a nonnegative integer $i$ with $i+1 \in \operatorname{dom}(x) \cap \operatorname{dom}(y)$ such that $x(i+1) \neq$ $1 \vee y(i+1) \neq 1$. This is handled exactly as in the corresponding case with the operator $T$ : if $k$ is the least such nonnegative integer, then $p(T x, T y) \leq \frac{1}{2} p(x, y)$ or $p(T x, T y) \leq \frac{1}{2} p(x, T x)$ according to whether $x(k+1) \neq y(k+1)$ or $x(k+1)=y(k+1)$, respectively.

Case 2. $x(i)=1$ for all $i \in \operatorname{dom}(x)$ and $x \subseteq y$. If $x \in X_{n}$, then $T_{1} x=x$, and so $p\left(T_{1} x, T_{1} y\right)=$ $\frac{1}{2^{n}}=p(x, x)$. If $x \in X_{\infty}$, then $x=y=s_{\infty}=T_{1} x$, so $p\left(T_{1} x, T_{1} y\right)=0$.

Case 3. $y(i)=1$ for all $i \in \operatorname{dom}(y)$ and $y \subseteq x$. This reduces to the previous case.

\section{Competing interests}

The authors declare that they have no competing interests.

\section{Authors' contributions}

All authors contributed equally and significantly in writing this paper. All authors read and approved the final manuscript.

\section{Acknowledgements}

This work was supported by Grant No. 174025 of the Ministry of Science, Technology and Development, Republic of Serbia.

Received: 18 July 2013 Accepted: 15 October 2013 Published: 19 Nov 2013

\section{References}

1. Ćirić, LB A generalization of Banach's contraction principle. Proc. Am. Math. Soc. 45, 267-273 (1974)

2. Ćirić, LB: Some recent results in metrical fixed point theory. University of Belgrade, Beograd (2003)

3. Rhoades, BE: A comparison of various definitions of contractive mappings. Trans. Am. Math. Soc. 226, $257-290$ (1977)

4. Matthews, SG: Partial metric topology. In: Proc. 8th Summer Conference on General Topology and Applications. Ann. New York Acad. Sci., vol. 728, pp. 183-197 (1994)

5. Abdeljawad, T, et al.: Existence and uniqueness of a common fixed point on partial metric spaces. Appl. Math. Lett. 24(11), 1894-1899 (2011). doi:10.1016/j.aml.2011.05.014

6. Abdeljawad, T, et al:: A generalized contraction principle with control functions on partial metric spaces. Comput. Math. Appl. 63(3), 716-719 (2012)

7. Abdeljawad, T, et al.: Coupled fixed points for Meir-Keeler contractions in ordered partial metric spaces. Math. Probl. Eng. 2012, Article ID 327273 (2012)

8. Altun, I, Sola, F, Simsek, H: Generalized contractions on partial metric spaces. Topol. Appl. 157(18), 2778-2785 (2010)

9. Aydi, $\mathrm{H}$, et al:: Berinde type generalized contractions on partial metric spaces. Abstr. Appl. Anal. 2013, Article ID 312479 (2013)

10. Aydi, H, Vetro, C, Sintunavarat, W, Kumam, P: Coincidence and fixed points for contractions and cyclical contractions in partial metric spaces. Fixed Point Theory Appl. 2012, 124 (2012)

11. Bukatin, M, Kopperman, R, Matthews, S, Pajoohesh, H: Partial metric spaces. Am. Math. Mon. 116, 708-718 (2009)

12. Chi, KP, et al.: A generalized contraction principle in partial metric spaces. Math. Comput. Model. 55(5-6), 1673-1681 (2012)

13. Ćirić, LB, Samet, B, Aydi, H, Vetro, C: Common fixed points of generalized contractions on partial metric spaces and an application. Appl. Math. Comput. 218, 2398-2406 (2011)

14. Di Bari, C, Kadelburg, Z, Nashine, HK, Radenović, S: Common fixed points of g-quasicontractions and related mappings in 0-complete partial metric spaces. Fixed Point Theory Appl. 2012, 113 (2012)

15. Di Bari, C, Milojević, M, Radenović, S, Vetro, P: Common fixed points for self-mappings on partial metric spaces. Fixed Point Theory Appl. 2012, 140 (2012)

16. Di Bari, C, Vetro, P: Fixed points for weak $\phi$-contractions on partial metric spaces. Int. J. Eng. Contemp. Math. Sci. 1(1), 5-13 (2011)

17. Erhan, M, et al.: Fixed point theorems for operators on partial metric spaces. Appl. Math. Lett. 24(11), 1900-1904 (2011). doi:10.1016/j.aml.2011.05.013

18. Karapinar, E: A note on common fixed point theorems in partial metric spaces. Miskolc Math. Notes 12(2), 185-191 (2011)

19. Karapinar, E: Generalizations of Caristi Kirk's theorem on partial metric spaces. Fixed Point Theory Appl. 2011, 4 (2011)

20. Nashine, HK, et al.: Fixed point results in orbitally complete partial metric spaces. Bull. Malays. Math. Sci. Soc. 36(4) (2013)

21. Oltra, S, Valero, O: Banach's fixed theorem for partial metric spaces. Rend. Ist. Mat. Univ. Trieste 36, 17-26 (2004)

22. Paesano, D, Vetro, P: Common fixed points in a partially ordered partial metric space. Int. J. Anal. 2013, Article ID $428561(2013)$

23. Paesano, D, Vetro, P: Suzuki's type characterizations of completeness for partial metric spaces and fixed points for partially ordered metric spaces. Topol. Appl. 159(3), 911-920 (2012) 
24. Rabarison, AF: Partial metrics. Supervised by Hans-Peter A. Künzi, African Institute for Mathematical Sciences (2007)

25. Rakočević, V, et al.: On cyclic generalized weakly C-contractions on partial metric spaces. Abstr. Appl. Anal. 2013, Article ID 831491 (2013)

26. Roldan, A, et al.: Multidimensional fixed point theorems in partially ordered complete partial metric spaces under $(\psi, \varphi)$-contractivity conditions. Abstr. Appl. Anal. 2013, Article ID 634371 (2013)

27. Romaguera, S: A Kirk type characterization of completeness for partial metric spaces. Fixed Point Theory Appl. 2010 Article ID 493298 (2010). doi:10.1155/2010/493298

28. Tas, K, et al.: Fixed point theorem on partial metric spaces involving rational expressions. Miskolc Math. Notes 14(1), 135-142 (2013)

29. Vaezpour, M, et al.: A common fixed point theorem for cyclic operators on partial metric spaces. Filomat 26(2), 407-414 (2012)

30. Vetro, C, Vetro, F: Common fixed points of mappings satisfying implicit relations in partial metric spaces. J. Nonlinear Sci. Appl. 6(3), 152-161 (2013)

31. Vetro, F, Radenović, S: Nonlinear $\psi$-quasi-contractions of Ćirić-type in partial metric spaces. Appl. Math. Comput. 219 1594-1600 (2012)

32. Bukatin, MA, Shorina, SY: Partial metrics and co-continuous valuations. In: Nivat, M (ed.) Foundations of Software Science and Computation Structure. Lecture Notes in Computer Science, vol. 1378, pp. 125-139. Springer, Berlin (1998)

33. Matthews, SG: An extensional treatment of lazy data flow deadlock. Theor. Comput. Sci. 151, 195-205 (1995)

34. Schellekens, MP: The correspondence between partial metrics and semivaluations. Theor. Comput. Sci. 315, 135-149 (2004)

35. Ilić, D, Pavlović, V, Rakočević, V: Some new extensions of Banach's contraction principle to partial metric space. Appl. Math. Lett. 24, 1326-1330 (2011)

36. Haghi, RH, Rezapour, S, Shahzad, N: Be careful on partial metric fixed point results. Topol. Appl. 160, $450-454$ (2013)

37. Hardy, GE, Rogers, TD: A generalization of a fixed point theorem of Reich. Can. Math. Bull. 16, $201-206$ (1973)

10.1186/1687-1812-2013-303

Cite this article as: Ilić et al.: Three extensions of Ćirić quasicontraction on partial metric spaces. Fixed Point Theory and Applications 2013, 2013:303

\section{Submit your manuscript to a SpringerOpen ${ }^{\circ}$ journal and benefit from:}

- Convenient online submission

Rigorous peer review

- Immediate publication on acceptance

- Open access: articles freely available online

- High visibility within the field

- Retaining the copyright to your article 